\title{
The epidemiology of cancer-related pain
}

\author{
*Escola de Enfermagem da Universidade de São Paulo. \\ **Ambulatório de Dor da Clínica Neurológica do Hospital das \\ Clínicas da Faculdade de Medicina da USP.
}

Epidemiology, when understood as the study of the distribution and determinants of disease in the population, has three key aspects: frequency, distribution and determinants. Not always the disease is the only relevant factor. At times, the deficiences or symptoms stemming from the pathology weigh in the altering of life quality to such an extent that they become the main point in the person's life. This is what happens in the case of cancer pain. It affects sleep, appetite, physical activity, hygiene, humor, interpersonal relationships, the ability to concentrate, sexual activity, as well as other aspects.

There are few, limited epidemiological studies on cancer-related pain that adress frequency of occurrence, characteristics, distribution, and determining factors. Nonetheless, studies carried out utilizing groups of patients demonstrate that the frequency with which pain occurs is of such magnitude that the pain control is one of the four priorities of the World Health Organization's (WHO) cancer control program, which considers this subject a public health problem.

Pain is the most feared oncology symptom. It may be acuteur chronic. When acute, it generally results from diagnostic or therapeutic actions. When chronic, it is related to the tumor or its metastases in about two-thirds of the cases by bone and neural invasion. Infiltration of the soft tissues, compression or occlusion of the blood vessels, and obstruction of hollow viscera. Aproximately one-fifth of the time pain is the consequence of antineoplastic therapies, such as those associated with actinic lesions. It may be due to increase in nociception stimulation (nociceptive pain), by lesion of the central or peripheral nerve pathways (deafferentation pain), and is frequently due to a combination of these. The occur- rence of cancer pain is due to the caracteristics of the disease itself and to the characteristics of the patient. It is related to the extesion of the disease, the presence of metastases, and the location of the tumor. The patient's cultural and affective aspects and the symbolic significance he attributes to pain also influence. In metastases or advanced loco-regional neoplasiasm between $40 \%$ to $95 \%$ of the patients feel pain 1 . In cases initiating and those without metastasis, frequency is between $11 \%$ and $75 \%$. In every phase of the illness, pain is felt by $50 \%$ of the patients. Traditionaly, pain is through to occur more in bone tumors when it also attacks organs such the pancreas, liver, and lungs, among others, and when the tumor is located in the cephalic segment, in contrast to the leukemias. It is highly possible that the size of the tumor and the involvement of sensitive nerve structures, more than the location of the tumor, has greater influence in the occurence of pain.

The influence of the affective state on pain exists. Pain and depression are associated and pain frequency is greater among depressed cancer patients than those who are not.

Intensity of cancer pain varies in accord with the location of the tumor, of the metastases, and the stage of neoplastic evolution, in adittion to variations related to the socio-cultural and psychological aspects of each person. Pain is moderate in between $30 \%$ to $66 \%$ of the cases and has been described as intense os excruciating 17.5 to $40 \%$ of the time. The most intense pains greatly affect daily life. Affective and cultural aspects influence in the magnitude of algetic symtomatology. Depressed patients undergo more intense pain as do those having misconceptions of cancer pain and analgesia. It has also been observed that patients who believe that pain is a signal of the disease's 
advance feel more intense pain than those who do not make this relation.

The majority of the patients feel pain in more than one place, and duration of the algetic symptom is related to the time the disease has been developing and the stage it is in, varying between days to years. There may be periods when the pain recurs when the therapies are begun, and returns in cases of recidivation of the disease or when it has not been controlled.

WHO has set forth guidelines for the control cancer pain: oral medication is preferred; the analgesic should be perscribed in a scheduled basis, not on demand, and should ascend the scale of potency gradually. This procedure should beginy first administering anti-inflammatory analgesics, associates or not with adjuvants (antidepressants, anticonvulsants, neuroleptics and corticosteroids). If pain control is not satisfactory, use of opiates, strong and weak, associated or not adjuvants, is initiated.

Despite recommendations, cancer pain has not been brought under sufficient control. Contributing to this are aspects related to the professionals involved, the patients, and the health system. Training of health professionals in cancer associated pain and analgesia is scant and results in the underestimation of its importance in the morbidity, and the impact on the biological, psychological, and social spheres of the patient. Moreover, there are a great number of professionals who are not knowledgeable in cancer pain control methods and have misconceptions concerning tolerance and physical and psychological dependence phenomena when opiates are used. The patients and their family members also share in these misconceptions, which can result in reluctance in reporting of the pain and adhering to the treatment.

The greater portion of the health system operates within a model to treat the disease. Consequently, control of the symptoms is not given priority. Legal and economic aspects are relative to the prescribing and sale of these medicines are barriers to the proper management of cancer pain. There is a lack of studies identifying risk groups undergoing inadequate pain control. Socio-cultural factors such as patients treated in centers attending ethnic minorities, senior citizens (over 70), woman, patients in better general health, and discrepancy between the physician's judgement and the patient's regarding the severity of the pain were indicative of insufficient control of painful symtomatology. 